\title{
Novel Spectral Kurtosis Technology for Adaptive Vibration Condition Monitoring of Multi Stage Gearboxes
}

Len Gelman ${ }^{* a}$, N. Harish Chandra ${ }^{\mathrm{a}}$, Rafal Kurosz ${ }^{\mathrm{a}}$, Francesco Pellicano ${ }^{\mathrm{b}}$, Marco Barbieri $^{\mathrm{b}}$ and Antonio Zippo ${ }^{\mathrm{b}}$

${ }^{a}$ School of Aerospace, Transport and Manufacturing

Cranfield University,

Bedford-MK43 OAL, United Kingdom.

Corresponding Author email: l.gelman@cranfield.ac.uk

${ }^{b}$ Department of Engineering “Enzo Ferrari”, University of Modena and Reggio Emilia, Via Vignolese 905/B, 41125 Modena, Italy

\section{Abstract}

In this paper, novel Wavelet Spectral Kurtosis (WSK) technique is applied for early diagnosis of gear tooth faults. Two variants of Wavelet Spectral Kurtosis technique called Variable Resolution WSK and Constant Resolution WSK are considered for diagnosis of the pitting gear fault. The gear residual signal obtained by filtering the gear mesh frequencies is used as the input to the SK algorithm. The gain obtained by using the wavelet SK techniques when compared to classical Fourier Transform (FT) based SK, is confirmed by estimating tooth wise Fisher's Criterion of diagnostic features. The final diagnosis decision is made by a three stage decision making technique based on weighted majority rule. The probability of the correct diagnosis is estimated for each SK technique for comparison. An experimental study is presented in detail to test the performance of the wavelet spectral kurtosis techniques and the decision making technique.

\section{Introduction}

Vibration diagnosis of local gear tooth faults is a problem addressed by many researchers. In past several years, higher order spectra based techniques were developed for fault diagnosis [1],[2]. Most of the developed techniques; focus on the non-stationary vibration signature generated due to tooth damage. Techniques based on demodulation of amplitude and phase [3], filtering techniques such as 
adaptive filtering [4], Short Time Fourier Transform (STFT), Wavelet Transforms [5] are widely reported for fault diagnosis application of gears. Along with the main fault diagnosis techniques such as demodulation, blind convolution and SK filtering techniques, supporting technologies for automatic and effective time synchronous averaging are developed [8]. The filtering of mesh harmonics from the time synchronous averaged signals will yield the gear residual signal [9]. Early fault diagnosis demands advanced the Spectral Kurtosis (SK) technique [6],[7],[9]. The classical STFT-based SK (CSK) depends on the window length used for the calculation of the STFT [14]. It is suggested that the STFT window size should be smaller than the distance between two impulses and larger than the length of one impulse response [14] . An inadequately short window may produce SK with poor spectral resolution and reduced level of details.

The SK can be used as filter for filtering gear residual signal to extract response corresponding to the fault. Recently, Wang and Liang [10] introduced Adaptive Spectral Kurtosis (ASK) technique for diagnosis of rolling element bearing faults. To obtain an optimal filter, Combet and Gelman [9] proposed a technique based on thresholding procedure applied to the SK estimate. As an alternative to the STFT, Sawalhi and Randall [11] estimated SK using the variable resolution wavelet transform. Sawalhi and Randall [11] applied wavelet spectral kurtosis for diagnosis of rolling element bearings. Huang et. al [12] have introduced Wavelet adaptive SK filtering technique for diagnosis of gearbox tooth faults. In this paper Huang et. al [12] applied Morlet wavelet based spectral kurtosis for gearbox diagnosis. However, the use of Time Synchronous Average (TSA) signal and gear residual signal were not reported.

In the present study, the Wavelet Spectral Kurtosis based optimal filter is selected based on thresholding procedure [9] and the advantages of Wavelet SK technique are confirmed by investigating vibration data from an industrial gearbox.

The key highlights of this paper are listed as follows,

1. A new technique called Constant Resolution Wavelet Spectral Kurtosis (CRWSK) is proposed.. Industrial studies are presented for early diagnosis of pitting gear fault using the CSK, the VRWSK and the CRWSK techniques. 
2. The gains obtained using both WSK techniques are investigated by a comparative study with respect to the CSK technique.

3. A novel decision making technique called weighted majority rule is applied for the first time for diagnosis of gear faults.

\section{The Variable Resolution Wavelet Spectral Kurtosis: Theoretical description}

The spectral kurtosis is interpreted as an adaptive technique used to determine the most suitable frequency band for extraction of the impact related non-stationary component of the signal. It was shown in [14] that the SK of the sum $r(t)=x(t)+$ $n(t)$, where $x(t)$ is the nonstationary component and $n(t)$ is the stationary Gaussian noise, can be related to the SK of the non-stationary part $x(t)$ by,

$$
K_{r}(f)=\frac{K_{x}(f)}{[1+\rho(f)]^{2}}
$$

where $K_{r}(f)$ is the spectral kurtosis of the sum $r(t), K_{x}(f)$ is the spectral kurtosis of the non-stationary component $x(t)$, and $\rho(f)=\frac{s_{n}(f)}{s_{x}(f)}$ the ratio of the power spectral densities of $n(t)$ and $x(t)$ reflecting the noise-to-signal ratio with respect to the frequency.

The Continuous Wavelet Transform (CWT) of signal $x(t)$ is described as,

$$
\operatorname{cwt}(s, \tau)=\frac{1}{\sqrt{|s|}} \int_{-\infty}^{\infty} x(t) \cdot \psi^{*}\left(\frac{t-\tau}{s}\right) d t
$$

where $s$ and $\tau$ are the scale and time translation parameters respectively, $\psi$ is the mother wavelet function.

The classical Complex Morlet mother wavelet function is given by,

$$
\Psi(t)=\frac{1}{\sqrt{\pi f_{b}}}\left(e^{j 2 \pi f_{c w} t}-e^{-f_{b}\left(\pi f_{c w}\right)^{2}}\right) e^{-t^{2} / f_{b}}
$$

where $f_{b}$ is the bandwidth parameter, $f_{c w}$ is the characteristic frequency of the mother wavelet function, and $j$ is the imaginary unit.

The Morlet wavelet can effectively capture both magnitude and phase information and has been found to be well adapted to the case of local fault detection in essentially non-stationary and non-linear signals [15].The wavelet based SK ensures that the optimal filter is estimated, exactly corresponding to the signal transient 
impulses. The Wavelet Spectral Kurtosis (WSK) is calculated based on the wavelet transform as shown in equation below,

$$
W S K_{x}(f)=\frac{S_{4, x}(f)}{S_{2, x}(f)^{2}}-2
$$

where $\left.S_{n, x}(f)=\left\langle|W P S(f, T)|^{n}\right\rangle,<.\right\rangle$ stands for time average operator and $W P S(f, T)=|W(f, T)|^{2}$ is the wavelet power spectrum of the signal.

Traditionally, the Morlet wavelet parameter $f_{b}$ is constant over the entire time and frequency plane. The VRWSK is estimated by keeping the $f_{b}$ parameter constant for all frequencies.

\subsection{The Constant Resolution Wavelet Spectral Kurtosis (CRWSK)}

It is known that the VRWSK gives increasingly better time resolution at higher frequencies, at the expense of poor frequency resolution at lower frequencies. As presented in the above section, the $f_{b}$ parameter of the Morlet mother wavelet is constant for the VRWSK. In general, to estimate the VRWSK, a reference frequency must be chosen to fix the frequency resolution or time resolution of the SK. On the other hand, using the proposed CRWSK technique, the frequency resolution is matched with chosen SK window size by varying the $f_{b}$ parameter of the mother wavelet.

To obtain the CRWSK, it is proposed that, $f_{b}$ parameter of the Morlet mother wavelet function is varied along the frequency domain to keep the constant WSK resolution. The reference frequency instead of being a constant value as in case of VRWSK technique, for CRWSK technique the reference frequency will be a variable. The CRWSK ensures that the optimal filter is estimated, exactly corresponding to the signal transient impulses for all frequencies. In the following section the diagnosis procedure implemented in this paper is presented in brief.

\section{SK based diagnosis technology}

The Wavelet Spectral Kurtosis diagnosis technology is presented as follows,

1. An automated time synchronous averaging technique [8] is implemented for estimation of the shaft speed and the TSA signal from the raw vibration data. 
2. The gear residual signal is estimated by removing the mesh harmonics from the TSA signal. Considering overlap between the TSA segments, several gear residual signals termed as realizations are obtained.

3. The SK is estimated by using the Wavelet transform for undamaged and damaged data for all the realizations.

4. After confirming the consistency of the SK estimated over all the realizations, by thresholding procedure an optimal SK Weiner denoising filter is obtained for each realization.

5. The SK optimal filter is applied to each realisation of the gear residual signal to obtain the SK residual signal. The envelope of the SK residual signal is considered as diagnostic fault feature. These features are converted to toothwise features.

6. The above procedure is implemented for both undamaged and damage data sets for same SK filtering threshold. The Fisher's Criterion is used to check the separation between the undamaged and damaged features.

7. The features are used for final diagnosis decision making, a technique based on weighted majority rule [13].Three stages namely anomaly detection, damage detection and diagnosis decision are used for decision making.

\section{Industrial Case study}

\subsection{Experimental Setup}

Test rig is a multistage stage industrial gearbox. The schematic of test rig is as presented in Figure 1(a). This test rig consists of two similar gearboxes (Gearbox A and $B$ ) which are connected back to back through a shaft. The helical gears are denoted as $Z_{1}, Z_{2}, Z_{3}$ and $Z_{4}$ with teeth $14,46,13$ and 54 respectively as shown in Figure 1(a). The speed corresponding to the three shafts (Shaft 1,2 and 3 ) is $20 \mathrm{~Hz}$, $6.09 \mathrm{~Hz}$ and $1.4 \mathrm{~Hz}$ respectively. The picture of Gearbox $\mathrm{A}$ is as shown in Figure 1 (b). As presented in Figure 1(b), tri-axial accelerometers A1 and A2 are mounted on the gearbox at locations closer to shaft 1 and shaft 2 respectively.

The vibration data is captured at different instances of time, before and after damaged. Total 9 vibration data sets are captured after 0 hours, 1 hour, 15hours, 18 hours, 34 hours, 40 hours, 58 hours and 60.5 hours and are denoted as run\#1 to 
run\#9 consecutively. The data corresponding to Run \#1, Run\#2 and Run\#3 represent the undamaged data sets. After 58 hours of operation, the gear teeth are visually investigated to identify the presence of damage. At that instant, the pitting on the pinion Z3 (as highlighted in Figure 1-a) was noticed. Thus the data for Runs \#8 and \#9 represent the damaged response of the gearbox. The relative pitting damage percentage estimates were calculated from the area of the damaged surface relative to the surface of the whole tooth. The data acquired between 15 to 58 hours i.e. run \#4 to run \#7 are vibration data sets corresponding to transition from undamaged to damaged conditions. The photograph of the damaged gear tooth is presented in Figure 1(c). Torque information was also noted for every run. Average level of torque noted was $\sim 900 \mathrm{~N} . \mathrm{m}$ for all the test runs.

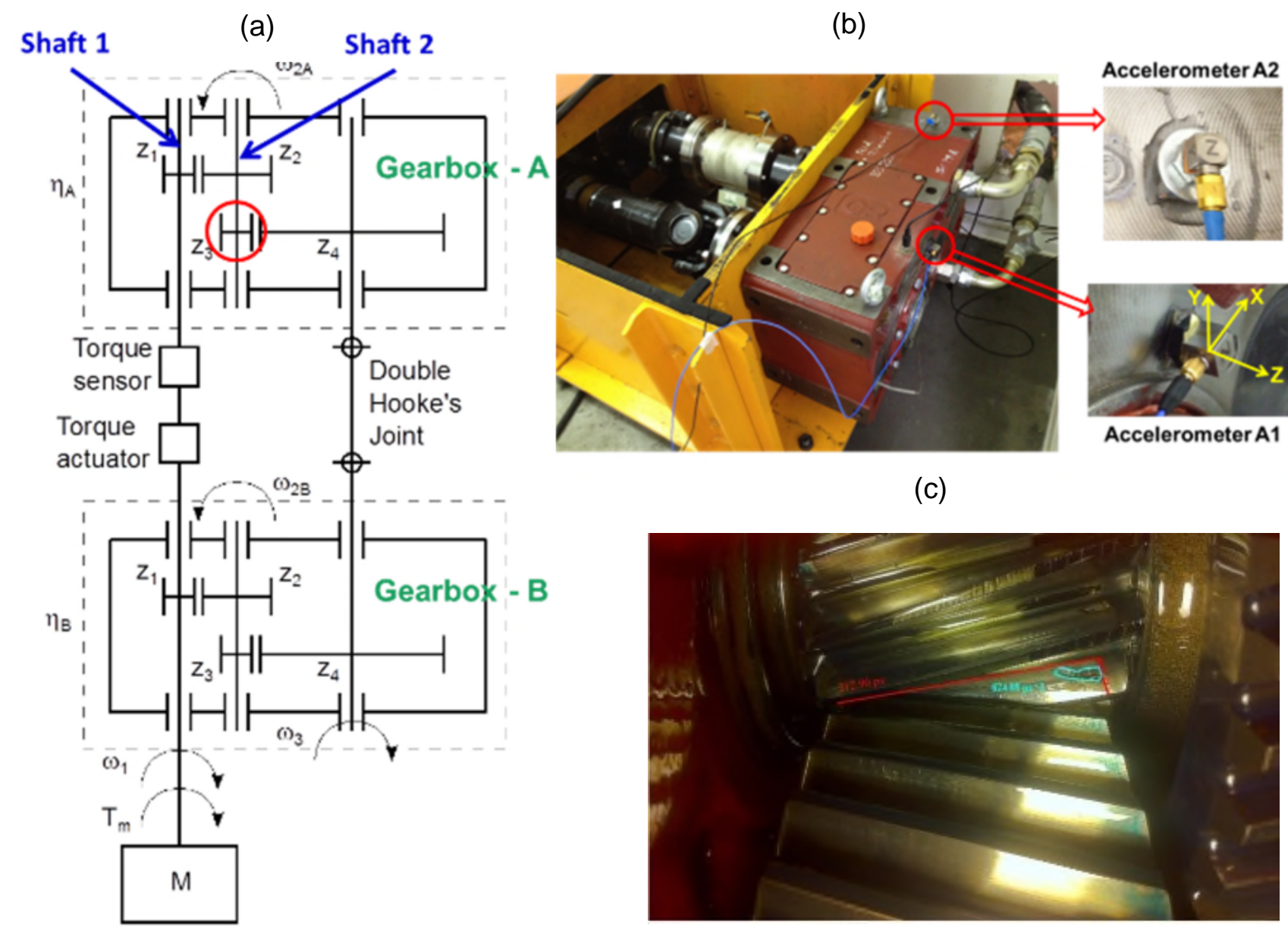

(b)

Figure 1 Test rig: Schematic Industrial gearbox with back to back gearbox connection

\subsection{Time Synchronous Averaging and Gear residual signal}

Prior to SK filtering, the raw vibration signal needs to be pre-processed in order to isolate vibrations related to individual components and remove interfering periodic 
components. Therefore, the SK is estimated by using the gear residual signal, which is obtained by resampling the vibration signal from time into the angular domain, time synchronous averaging and cleaning of periodic gear mesh components.

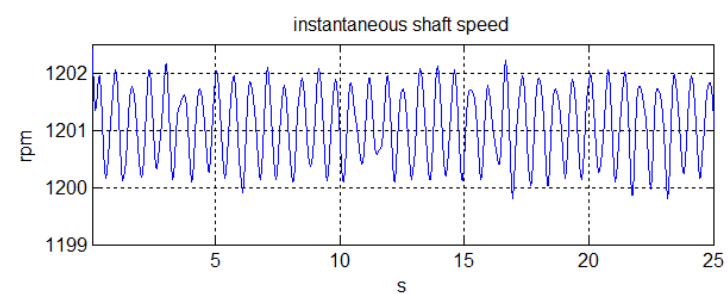

(a)

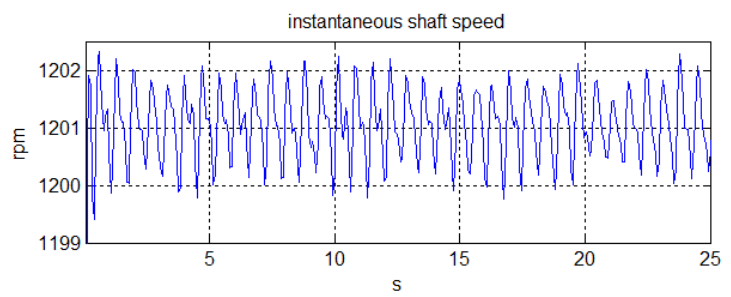

(b)

Figure 2 Estimates of the instantaneous rotation speed using automated technique (a) Run \#3 and (b) Run \#8

The input speed is accurately estimated as $\sim 1200 \mathrm{rpm}$ for both undamaged and damaged vibration data as shown in Figure 2. The TSA signal and its corresponding frequency spectrum are as presented in Figure 3.
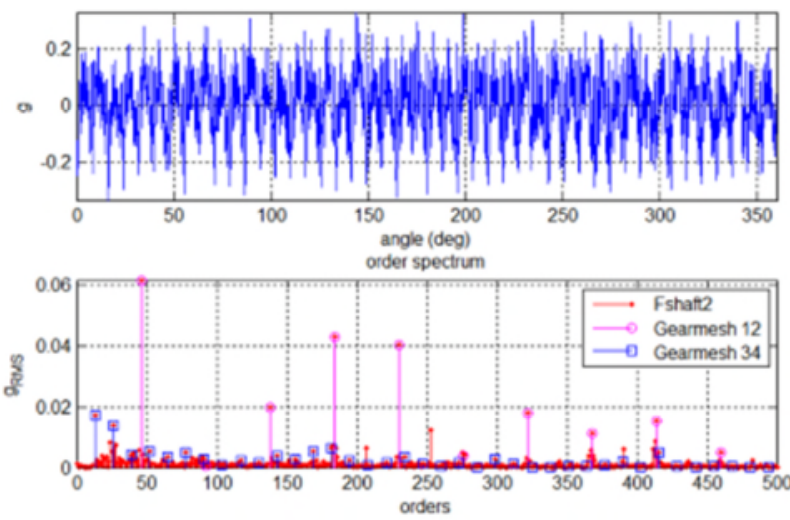

(a)
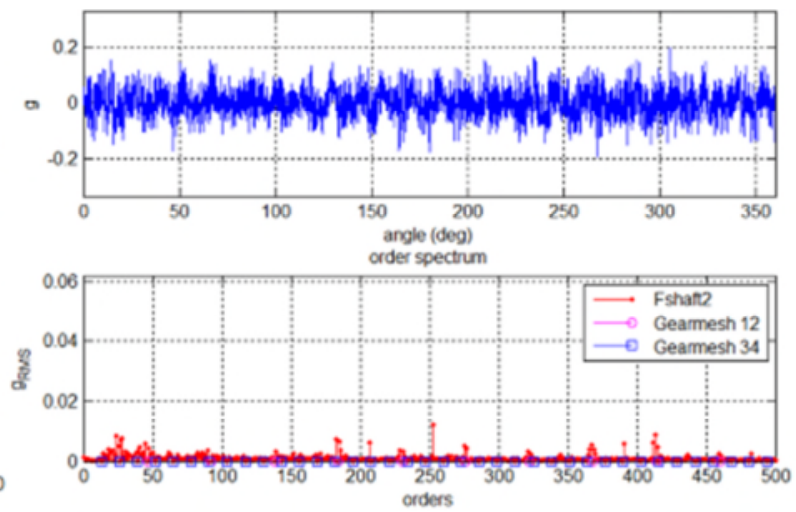

(b)

Figure 3 Response and its order spectrum (a) Time synchronous averaged (TSA) (b) Gear residual signal

In Figure 3, for the frequency spectrum of TSA and gear residual signals, the gearmesh12 refers to GMF corresponding to $Z_{1}$ and $Z_{2}$ gears; similarly gearmesh 34 refers to GMF corresponding to $Z_{3}$ and $Z_{4}$. It can be observed from Figure $3(a)$, the spectrum of the TSA response contains the GMF components corresponding to both stages of the gearbox. By filtering the gear mesh frequencies as shown in Figure 3 (b-bottom) and reconstructing using inverse Fourier transform the gear residual is obtained. Thus the gear residual clearly contains no spectral components 
corresponding gear mesh frequencies. It contains information corresponding to damage.

\subsection{Wavelet Spectral Kurtosis based optimal filtering}

The gear residual signal estimated for different TSA realizations is processed with classical and wavelet based spectral Kurtosis techniques. In this section the description for proposed WSK technique applied to test rig data is presented in detail. Later in this section, a comparative study between Classical Spectral Kurtosis (CSK) and Wavelet Spectral Kurtosis (WSK) is presented.

\subsubsection{Wavelet SK window size or resolution selection}

The frequency resolution corresponding to $1 \times$ GMF represents a window size equal to one mesh period. We considered the frequency resolution to be approximately equal to multiples of $2 / N_{w}$ (where $N_{w}$ is the length of the SK window). Thus in this paper, frequency resolution equal to $2 \times \mathrm{GMF}=158 \mathrm{~Hz}$ is termed as a full mesh time resolution and resolution of $4 \times \mathrm{GMF}=316 \mathrm{~Hz}$, is termed as half mesh time resolution.

In Figure 4, the WSK estimated for full and half mesh resolution for undamaged and damaged runs are presented. For variable resolution case two extra frequency bands were observed clearly.

\subsubsection{WSK estimation over realization and threshold}

By considering overlapping between TSA segments, 5 realizations for undamaged data and 18 realizations for damaged data are considered for estimating the Wavelet Spectral Kurtosis. The SK frequency band which represents the damage should be consistent over all the realizations.

As shown in Figure 5(a), for the undamaged run \#3, the techniques could not identify any significant and consistent frequency band which represents the damage. However, for the damaged case consistent frequency band with centre frequency $\sim 767 \mathrm{~Hz}$ is observed for both Wavelet SK techniques as shown in Figure 5. 

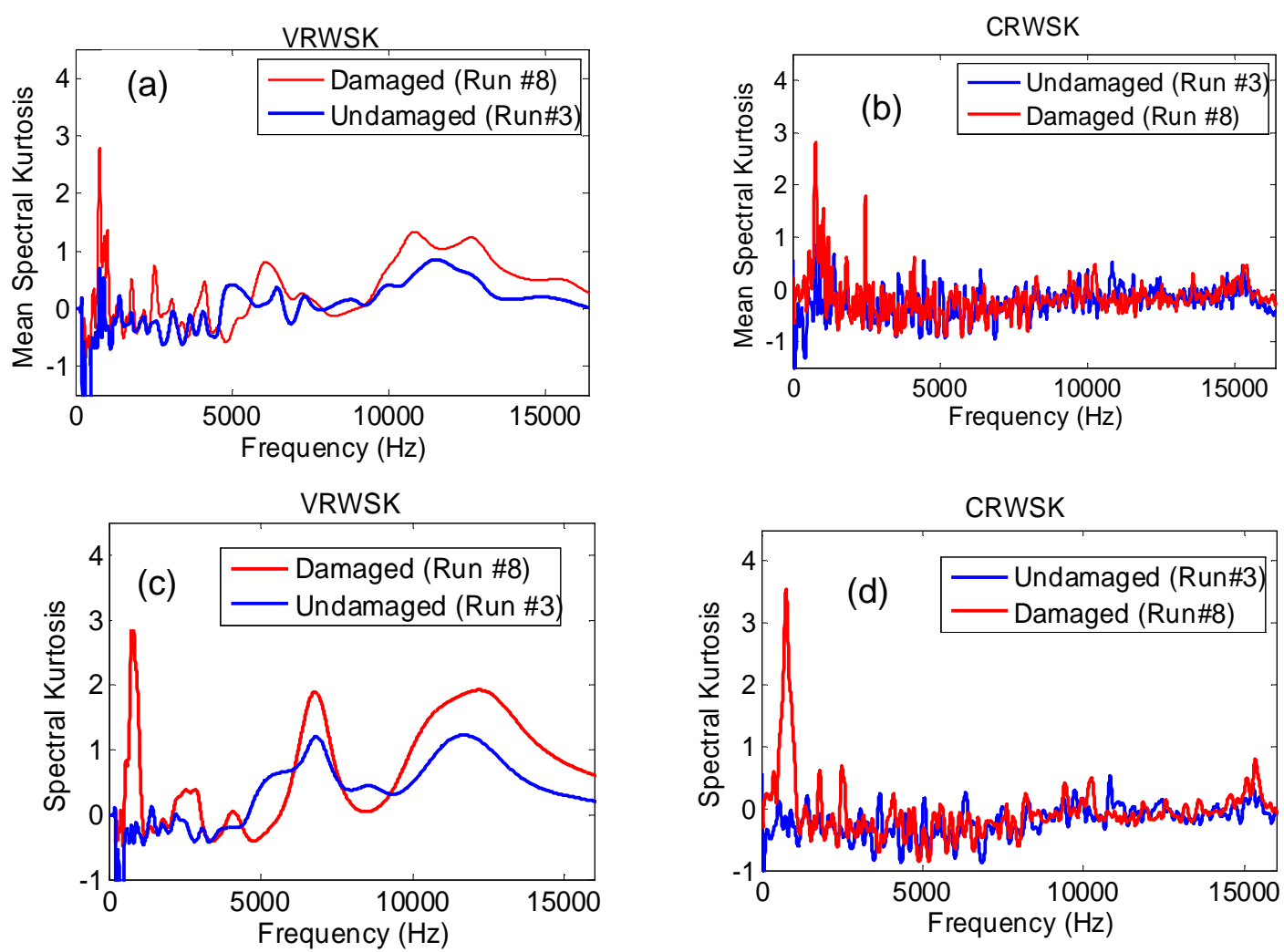

Figure 4 WSK for undamaged and damaged data (a) full mesh resolution SK using VRWSK technique (b) ) full mesh resolution SK using CRWSK technique, (c) half mesh resolution SK using VRWSK technique and (d) half mesh resolution SK using CRWSK technique

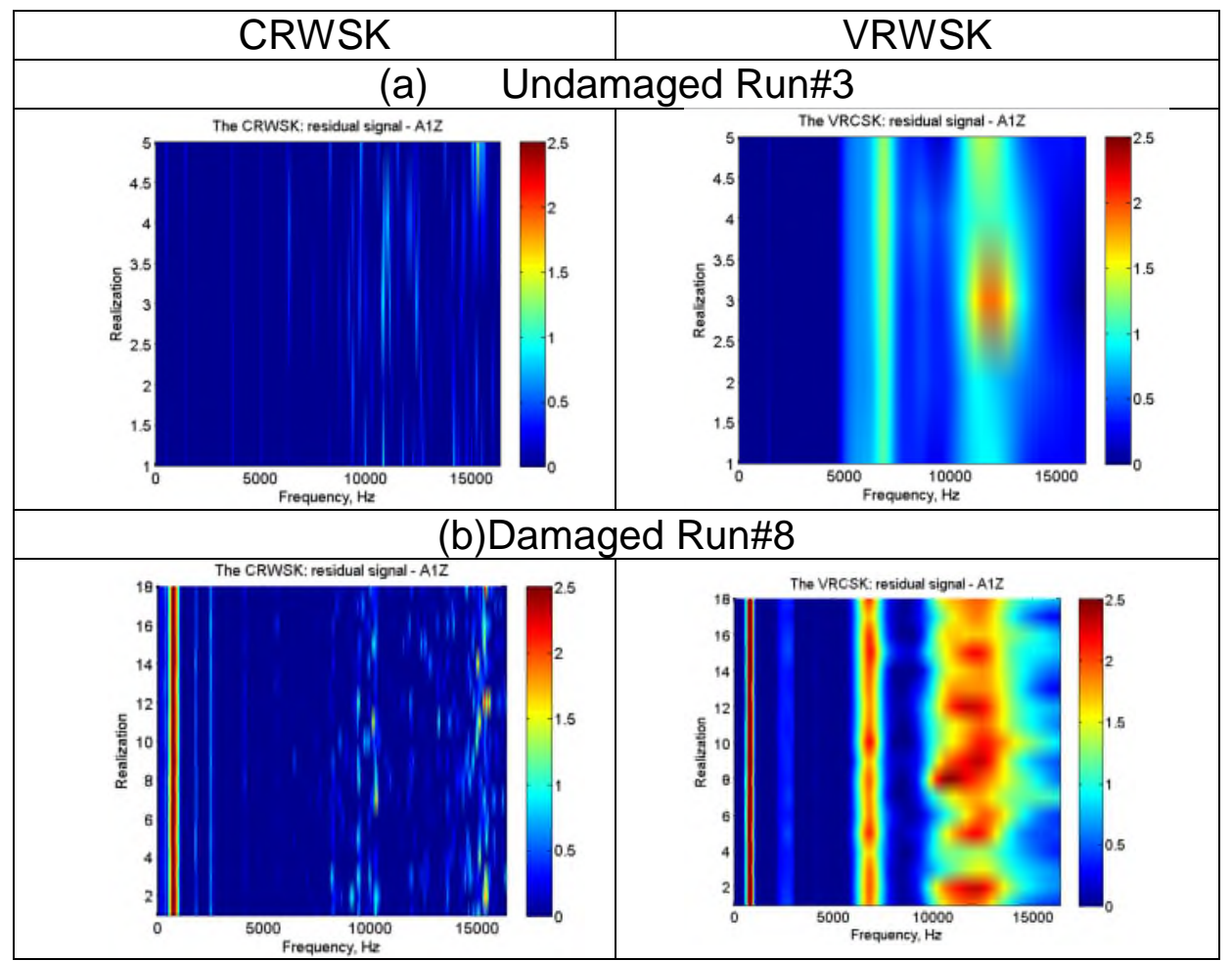

Figure 5 Spectral Kurtosis estimates for different realizations for undamaged (Run \#3) and damaged (Run \#8) data 


\subsubsection{SK based Optimal Filtering}

The Spectral Kurtosis (SK) was estimated from 18 consequent realizations of the gear residual signal obtained after removing mesh harmonics from the TSA signal. The window size corresponding to a half mesh period was used for SK calculation and the Weiner filters are as presented in Figure 6 . The SK for the damaged case has clearly expressed frequency band around frequency $\sim 767 \mathrm{~Hz}$ with $\mathrm{SK}>2.4$, where the SK for undamaged case remains very low for all the realizations.

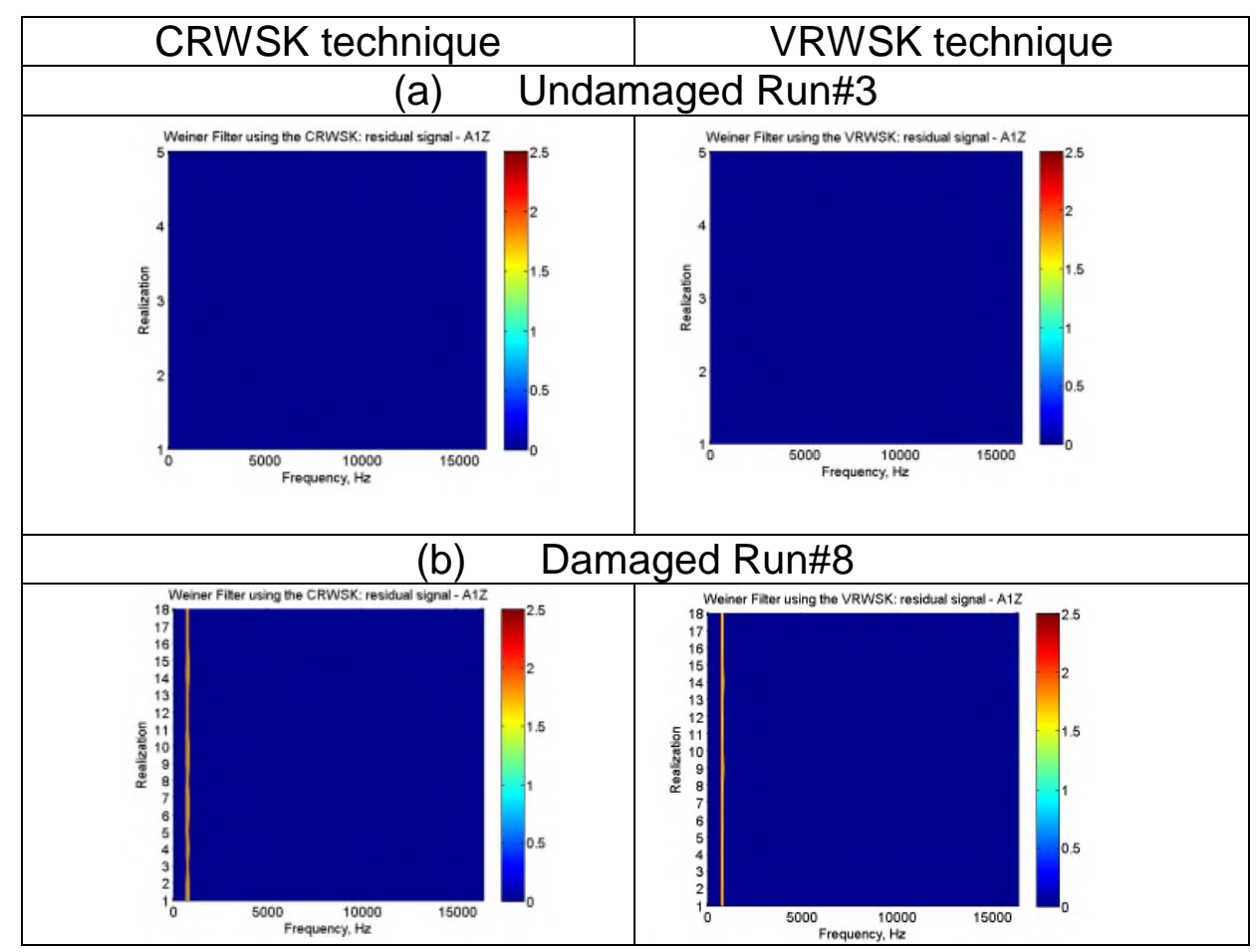

Figure 6 Spectral Kurtosis estimates for different realizations for undamaged Run\#3 and damaged Run \#8

The SK threshold $s=2.4$ is considered to obtain optimal denoising Wiener filter. SK filtering with threshold 2.4 on undamaged data did not show consistent SK frequency band for both the Wavelet Spectral Kurtosis techniques. On the contrary, the filtering using SK >s obtained from the damaged case resulted in removal of the stationary noise from the gear residual signal but retained the non-stationary component related to impulsive excitation due to a presence of the damage.

The next step in SK based diagnosis is to estimate the features using the SK residual signal. The SK residual signal is obtained by applying the optimal filters to 
the gear residual signal. The SK residual signal represents the vibration response corresponding to the frequency bands presented by the SK technique. The SK residual signal is transformed into angular domain based on the order (M) estimated using the TSA technique. Then the features in the angular domain are converted into toothwise SK features. The tooth wise SK features corresponding to the optimal filters presented in Figure 6 are as shown in Figure 7. Clearly, the damaged features are higher in magnitude when compared to undamaged features.

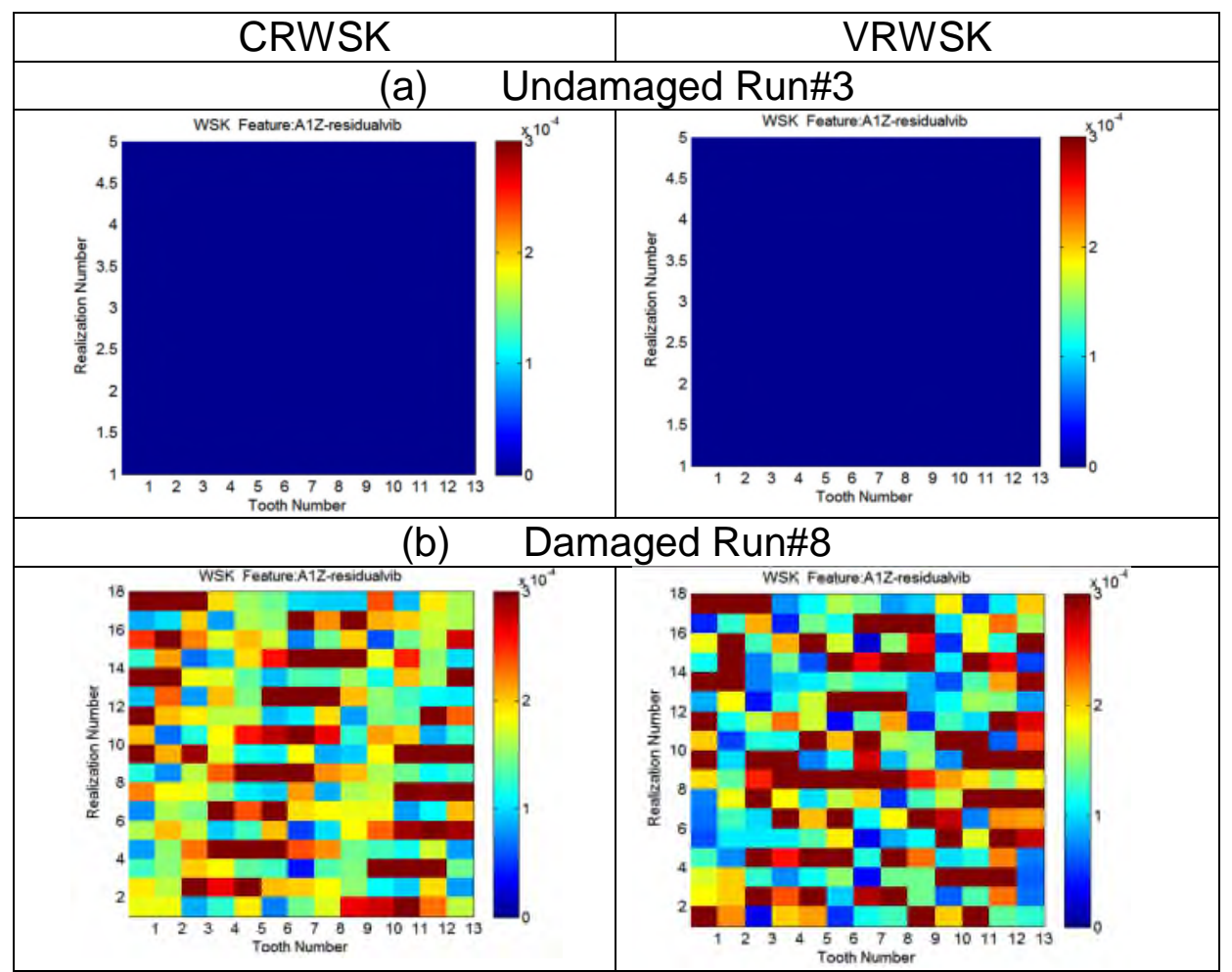

Figure 7 Toothwise SK features for half mesh SK resolution for undamaged Run\#3 and damaged Run \#8 for SK threshold =2.4

\subsection{Comparison of Wavelet SK with Classical Spectral Kurtosis}

The CSK estimate, the CSK Weiner filters and fault features for undamaged and damaged cases are presented in Figure 8. To compare the proposed Wavelet Spectral Kurtosis techniques with Classical SK technique in this section SK results corresponding to four frequency resolution choices are presented. 


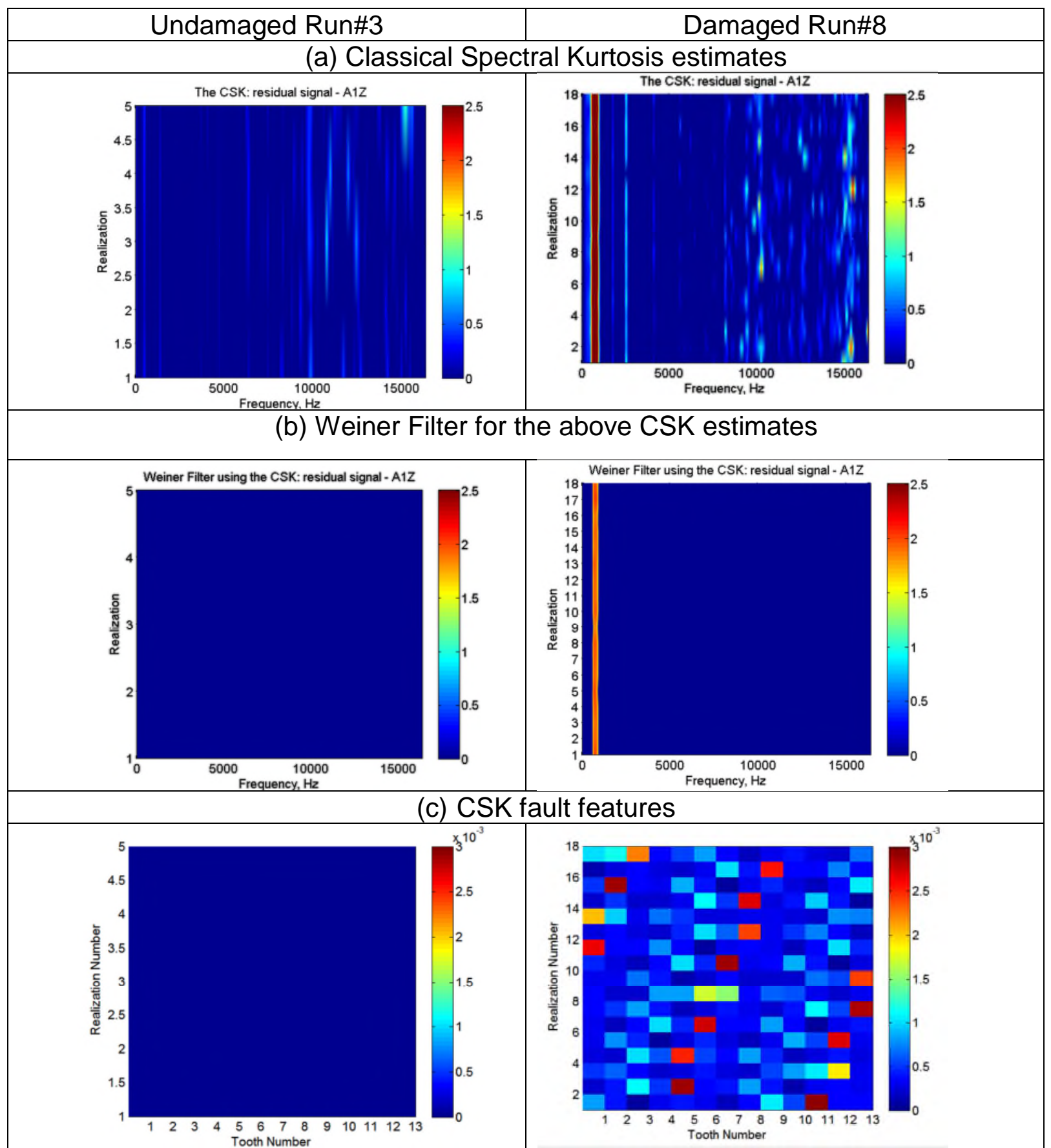

Figure 8 Classical Spectral Kurtosis results for half mesh $4 \times \mathrm{GMF}=317 \mathrm{~Hz}$

(a) CSK estimate (b) Weiner Filter estimate and (c) CSK fault features

As presented in Figure 9 ( $a$ and $b$ ), all the three SK techniques could not reveal a clear frequency band which indicates the presence of damage. For $4 \times G M F$ resolution as presented in Figure 9(e and f), all the three techniques consistently revealed presence of a single frequency band with centre frequency $\sim 767 \mathrm{~Hz}$ for damaged run \#8, while the undamaged SK did not show any consistent frequency 
band. On the other hand the wavelet spectral kurtosis depicted three frequency bands as shown in Figure $9(\mathrm{f})$. The first frequency band $\sim 767 \mathrm{~Hz}$ is generated due to the presence of impacts generated due damage. Any consistently occurring even as obtained at $\sim 767 \mathrm{~Hz}$ clearly indicates a damaged condition. The consistence of the SK estimate is verified by considering the SK estimate realization wise as shown in Figure 6. As presented in Figure 9(h), the impacts generated by the gears are clearly indicated by the use of the CRWSK technique. The resolution $13 \times$ GMF is considered to cover all the impacts generated by damaged pinion $\left(Z_{3}\right)$ with 13 teeth. For $13 \times$ GMF resolution as shown in Figure 9(h), the Classical Spectral Kurtosis frequency band at $\sim 767 \mathrm{~Hz}$ is very low when compared to the CRWSK and VRWSK techniques. 


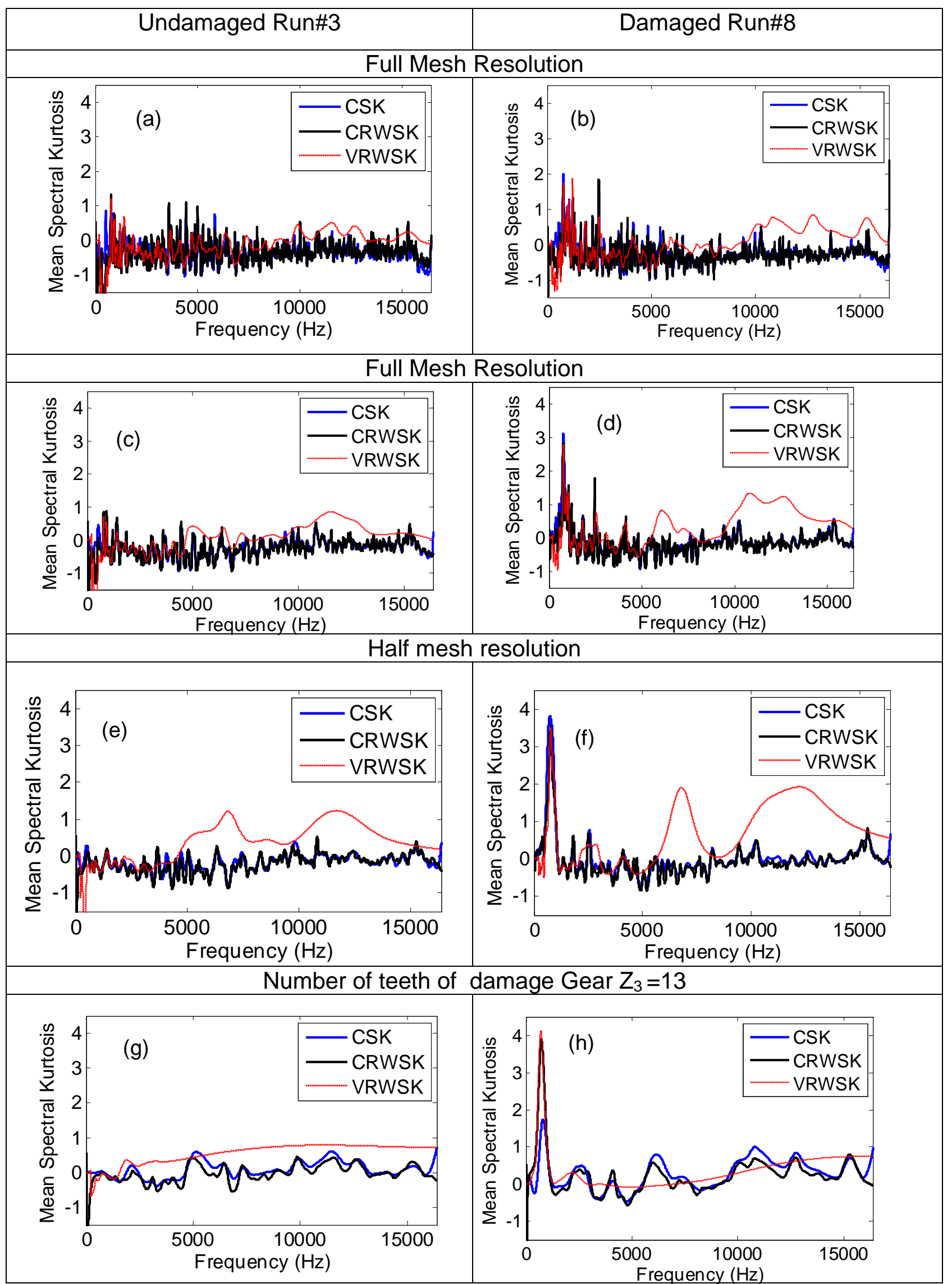

Figure 9 CSK, VRWSK and CRWSK estimates for undamaged and damaged runs for four different resolution values. 


\subsubsection{Advantage of Constant Resolution WSK technique}

The CRWSK technique has an advantage over variable resolution WSK technique as well as CSK technique. The description is presented as follows. As presented in section Error! Reference source not found., for the computation of the wavelet transform, a reference frequency, at which a fixed resolution is desired, should be given as an input for VRWSK technique. For CRWSK and CSK techniques the reference frequency input is not necessary, since using the CRWSK technique the resolution of the wavelet will adapt itself for every frequency step. A clear frequency band at $\sim 767 \mathrm{~Hz}$ as centre frequency is observed for the CRWSK technique. For the undamaged data, the frequency band of $\sim 767 \mathrm{~Hz}$ is not observed. Thus, this frequency is the unique frequency which represents the damaged case. Using the VRWSK technique if the reference frequency is equal to the SK frequency $(767 \mathrm{~Hz})$ as shown in Figure 10(a), VRWSK the SK curve shows a significant peak at this frequency. For reference frequencies other than $767 \mathrm{~Hz}$, using the VRWSK as presented in Figure $10(b, c)$, the frequency band at $\sim 767 \mathrm{~Hz}$ is not consistent.

Considering a complex system such as an industrial gearbox presented in this paper, the reference or the resonance frequency is difficult to estimate accurately. In such cases the resonance frequency depicted by the CSK technique can be used as reference frequency for VRWSK technique. Thus CRWSK can be used independently for diagnosis. The main disadvantage of VRWSK is that it cannot provide constant SK time resolution (i.e. full mesh resolution, half mesh resolution, etc.) for all frequencies, therefore, if only few teeth (one-two, three, etc) are damaged, then the VRWSK will not locate impacts from these teeth for all frequencies and as a result, the estimated SK will be perform with errors at some frequencies. In contrast, the CRWSK provides constant SK time resolution (i.e. mesh resolution, half mesh resolution, etc.) for all frequencies and as a result, estimation of the SK will be perform without errors for all frequencies. 
(a)

(b)

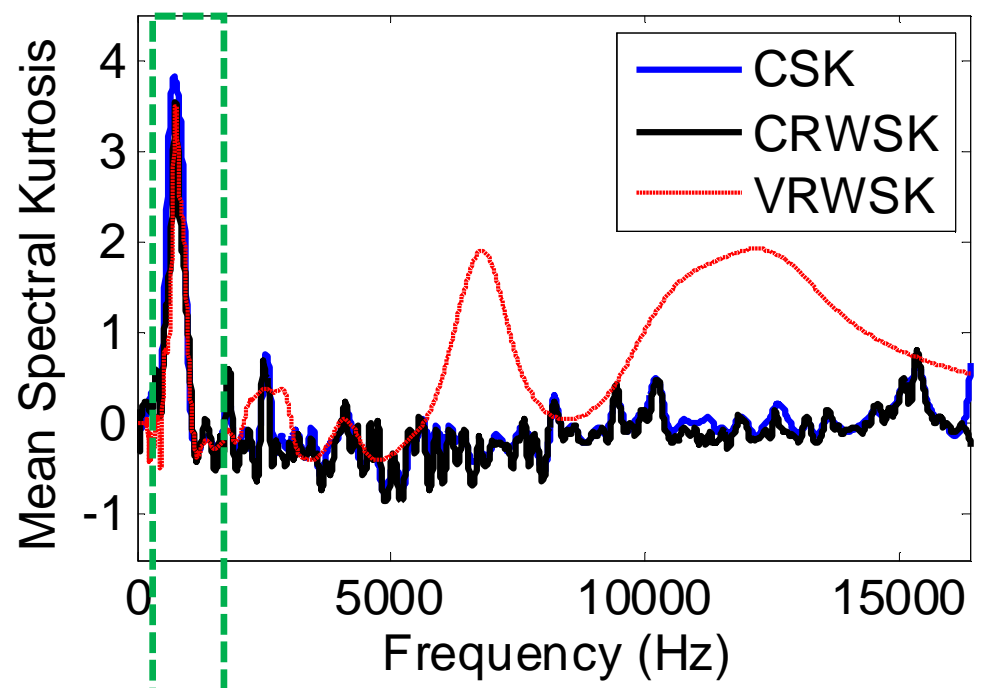

(c)
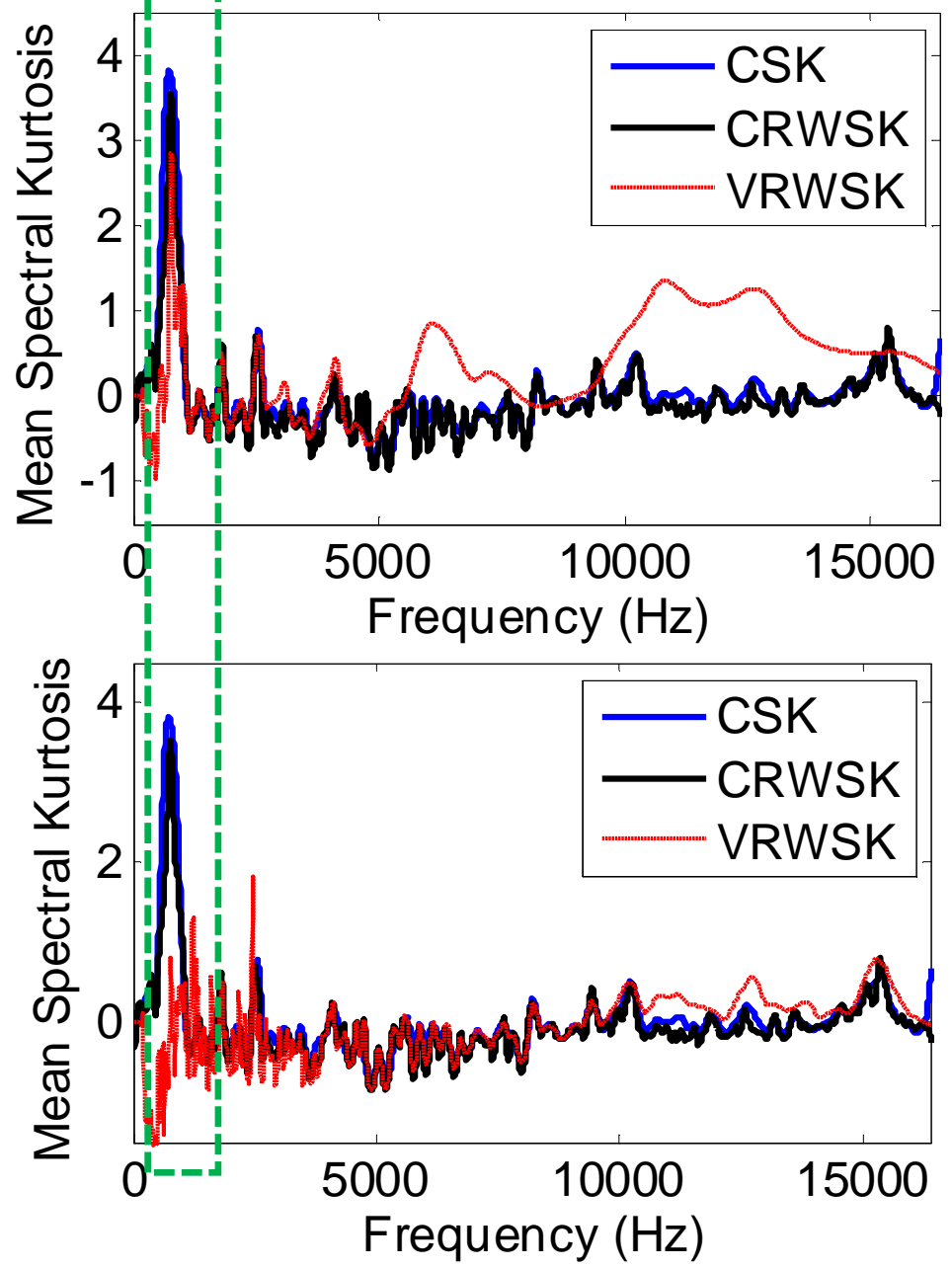

Figure 10 Spectral Kurtosis estimated using three techniques for different reference frequency of the wavelet transform 


\subsubsection{Comparison of SK features by Fisher's Criterion}

To compare the performance of CSK and WSK techniques gain parameter based on Fisher's Criterion (FC) is formulated [2]. Fisher's Criterion is a statistical parameter used to measure the separation between the SK features of undamaged and damaged data. The FC is given by the following equation.

$$
F C=\frac{\left(\mu_{p}-\mu_{u}\right)^{2}}{\sigma_{p}^{2}+\sigma_{u}^{2}}
$$

Where $\mu$ and $\sigma$ are the mean and standard deviation of the SK features, subscript $p$ represents pitting or damaged data case and subscript $u$ represents undamaged or no pitting data case. The tooth wise gain estimated as the ratio of the Fishers criterion (FC) by WSK to that of the FC obtained by CSK, which is given by GAIN = $\frac{F C_{W S K}}{F C_{C S K}}$. As shown in Figure 11(a), the Fisher's Criterion gain is significant for VRWSK technique. For the CRWSK technique the Fisher's Criterion estimated is same as the CSK technique for reference frequency $767 \mathrm{~Hz}$. As shown in Figure 11(c), for reference frequency $1500 \mathrm{~Hz}$, the CRWSK projected high separation between the damaged and undamaged data sets when compared to VRWSK technique. 


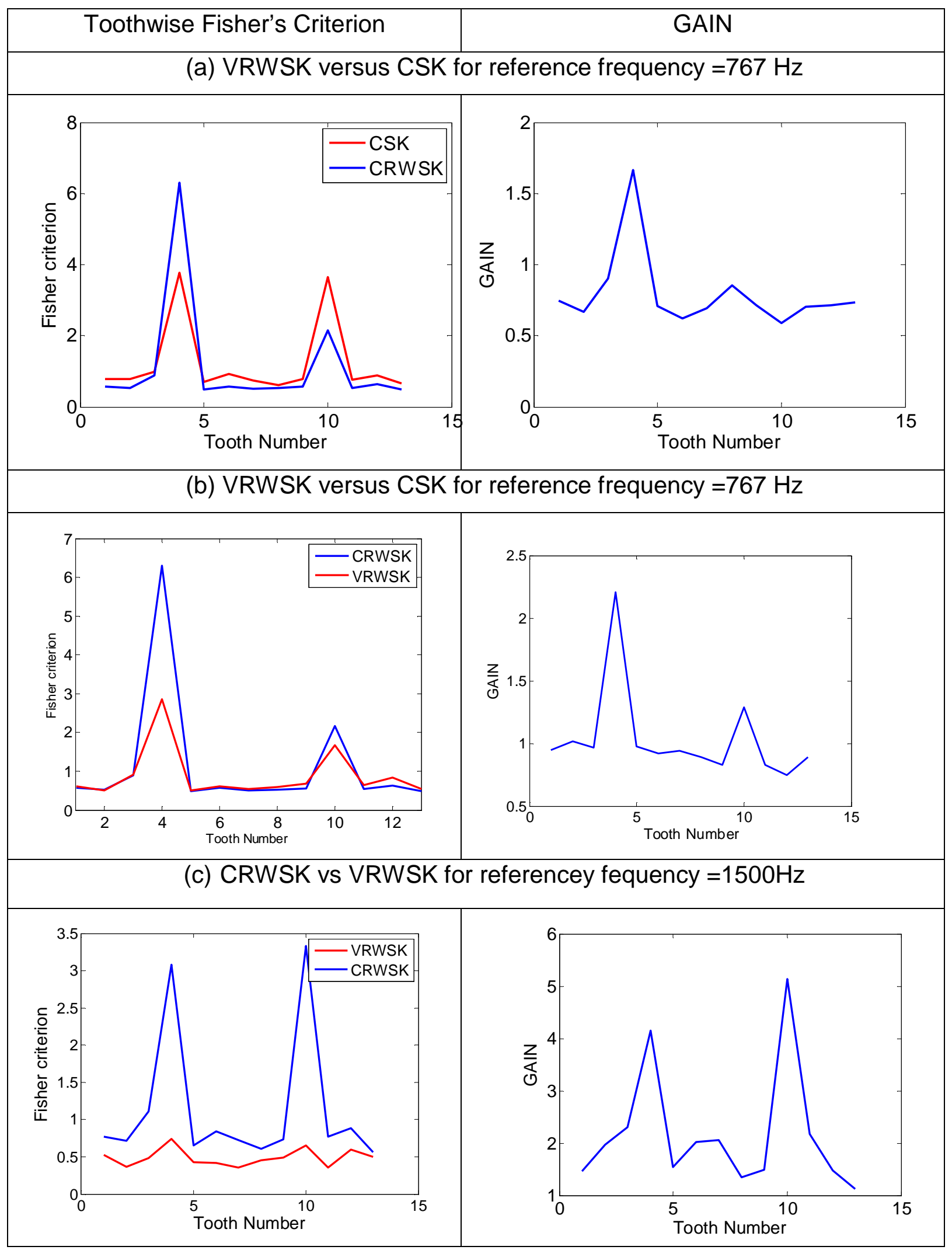

Figure 11 Fishers Criterion estimates for CSK and WSK techniques for Half mesh resolution 


\subsection{Decision making based on Weighted Majority Rule}

The implemented decision making method is the classification method based on the modified non-parametric kNN approach. The implemented method consists of the following preliminary steps:

- Data Clusterization,

- Calculation of the novelty scores,

For training purposes, the data were prepared by extracting the features from the undamaged case. This is followed by establishment of the training clusters using the k-means method, and calculation of the averaged distances to $k$ nearest neighbours (the averaged kNN distance) for each sample in each cluster of the training data. Thus, the maximal kNN distance for each fault-free training cluster, is estimated and used to establish the boundary between the fault-free and faulty clusters. The classical k-means algorithm was chosen for data clusterization; this algorithm creates compact clusters at low computational cost [19]. Calculation of the novelty scores for testing datasets, which contain diagnostic features independently for each tooth, is performed on the basis of the kNN algorithm, which concludes with calculation of a relative distance measure (i.e. the novelty score) used for final decision making. Decision-making procedure is based on the comparison of novelty scores with a detection threshold. The test data sample is believed to be the single anomaly when all novelty scores exceed the distance threshold, otherwise it is believed that no anomaly detected.

The SK features corresponding to Run\#2 and Run\#3 are chosen for training purpose. The decision making results corresponding to the undamaged data are presented in Figure 12 below. The training data is classified into 6 numbers of clusters and a kNN value of 7 is chosen for this study. In the anomaly detection stage of the undamaged case as shown in Figure 12, no false alarms were observed; these false alarms if present are eliminated in the damage detection and damage diagnosis stages. As shown in Figure 13, for the anomaly detection stage less nonanomalous detections were observed. By grouping these detections and by applying weighted majority rule as explained in section 2, the damage detection matrix is realized. The grouping procedure is repeated again on the damage detection matrix 
to obtain the final damage diagnosis matrix which is used for diagnosis decision making. As the separation between the undamaged and the damaged SK features is very high all the three SK techniques were successful in diagnosis. For SK Threshold $_{\text {I }}$ $=2.4$, the probability of correct diagnosis for CSK and CRWSK technique is $100 \%$. Using the VRWSK technique, the probability of correct diagnosis is $99 \%$. The decision making results for CSK, VRWSK and CRWSK techniques are presented in Figure 12, Figure 13 and Figure 14 respectively.

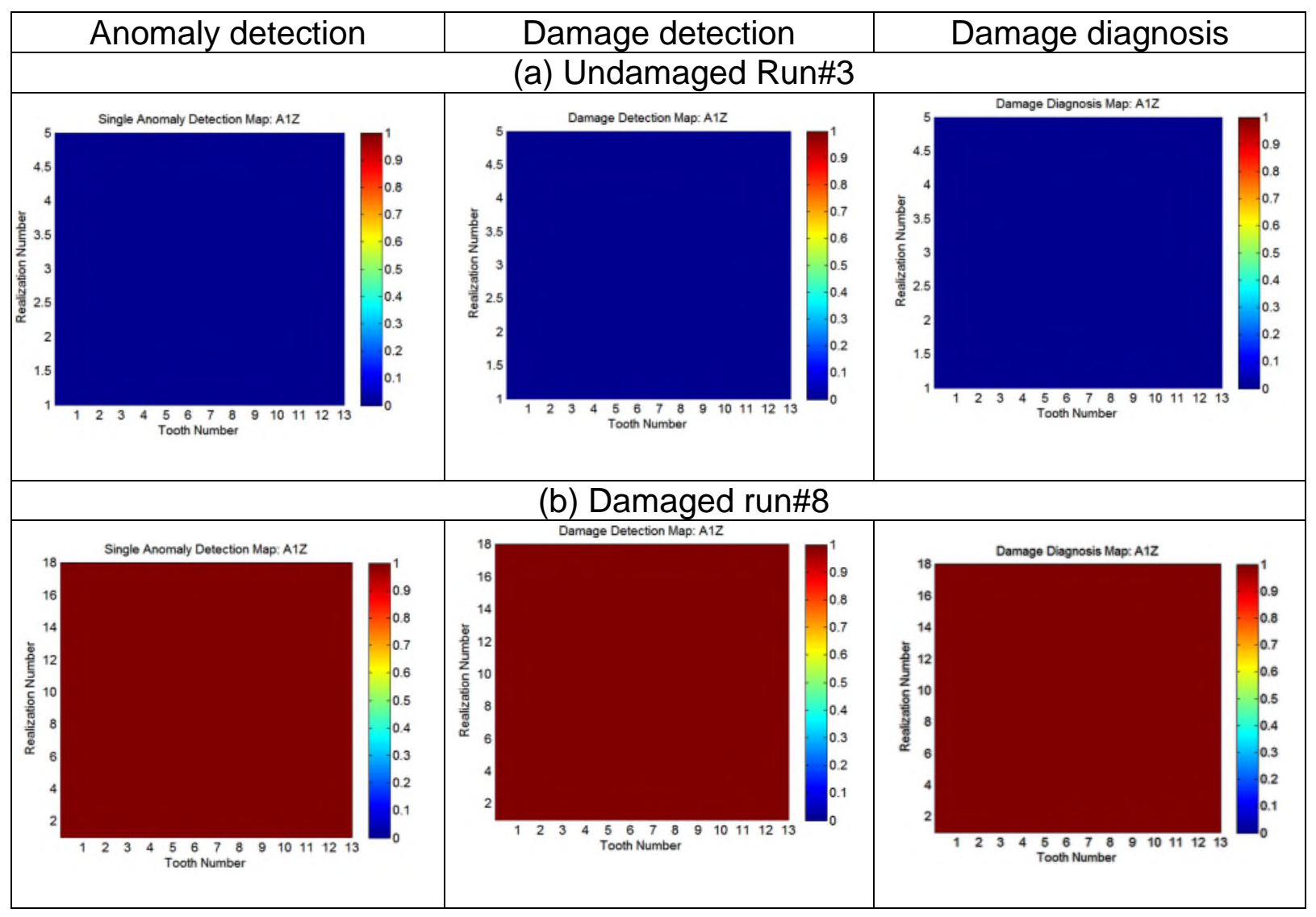

Figure 12 Decision making using CSK technique for undamaged (Run\#3) and damaged (Run\#8) 


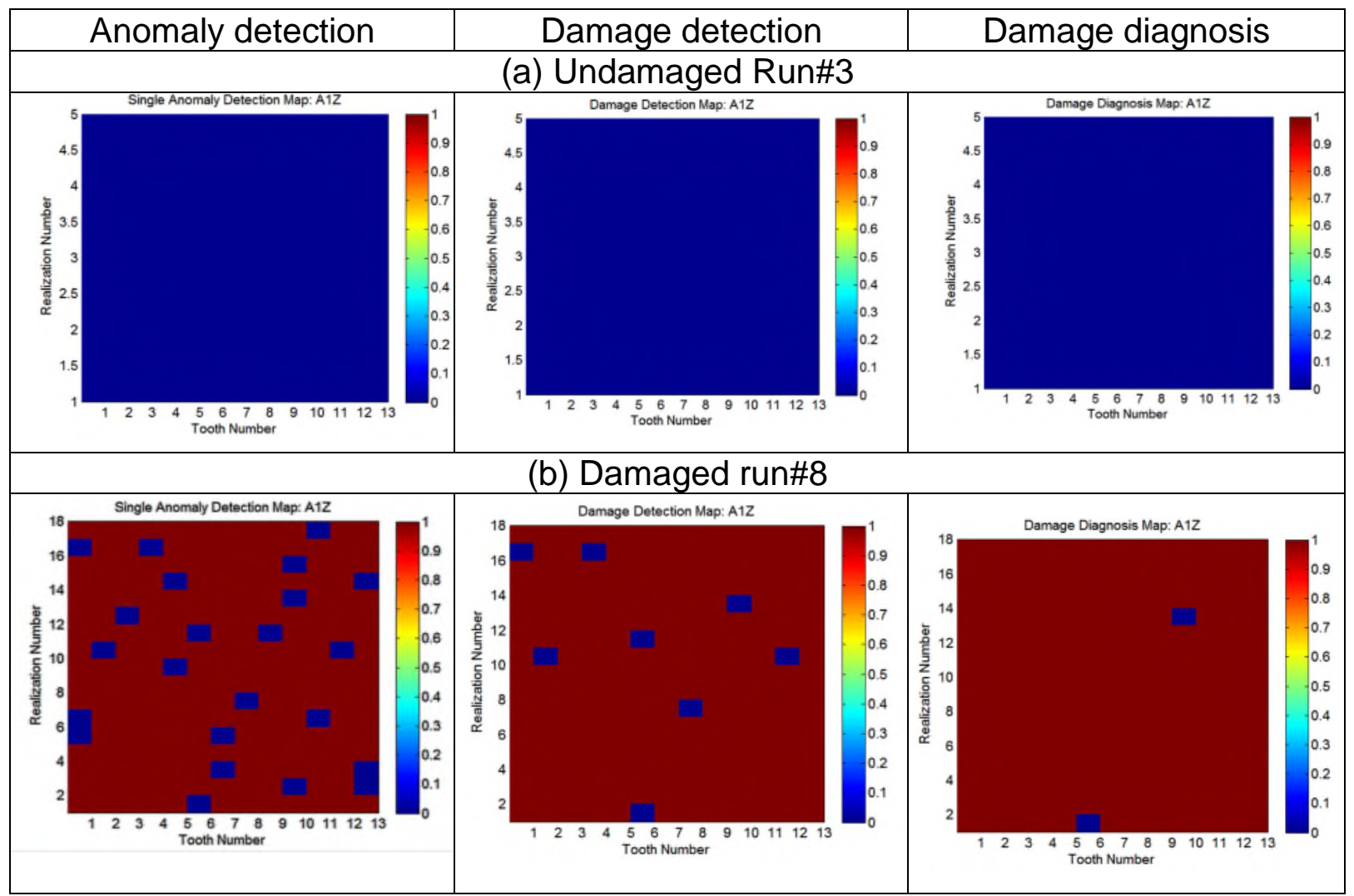

Figure 13 Decision making using VRWSK technique for undamaged (Run\#3) and damaged (Run\#8)

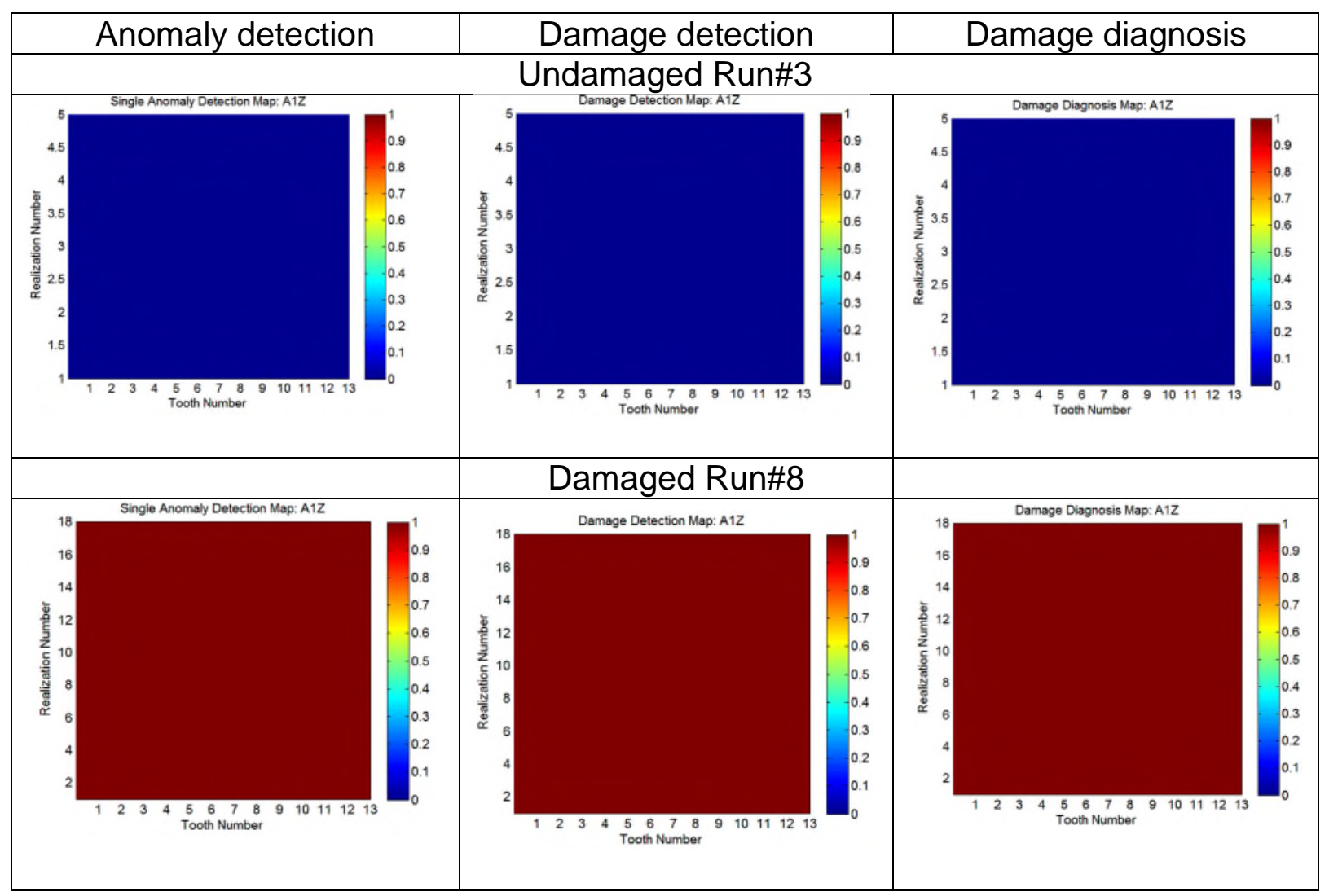

Figure 14 Decision making using CRWSK technique for undamaged (Run\#3) and damaged (Run\#8) 


\subsubsection{Performance evaluation of Wavelet Spectral Kurtosis by noise addition} As presented earlier the fisher's criterion has shown gain for VRWSK technique because of high separation between the SK features estimated with respect the CSK fault features. It is proposed to add noise check the effectiveness of the proposed technique. The signal to noise ratio is added using the below equation

$$
S N R=10 \log \left[\frac{\sigma_{s}^{2}}{\sigma_{n}^{2}}\right]
$$

where $\sigma_{n}^{2}$ indicates the variance of the noise response and $\sigma_{s}^{2}$ represents the variance of the signal. For a desired SNR, and known $\sigma_{\mathrm{s}}$, the added noise response is $s(t)=\sigma_{n} R_{p}$, where $R_{p}$ is a random parameter with normal distribution. 


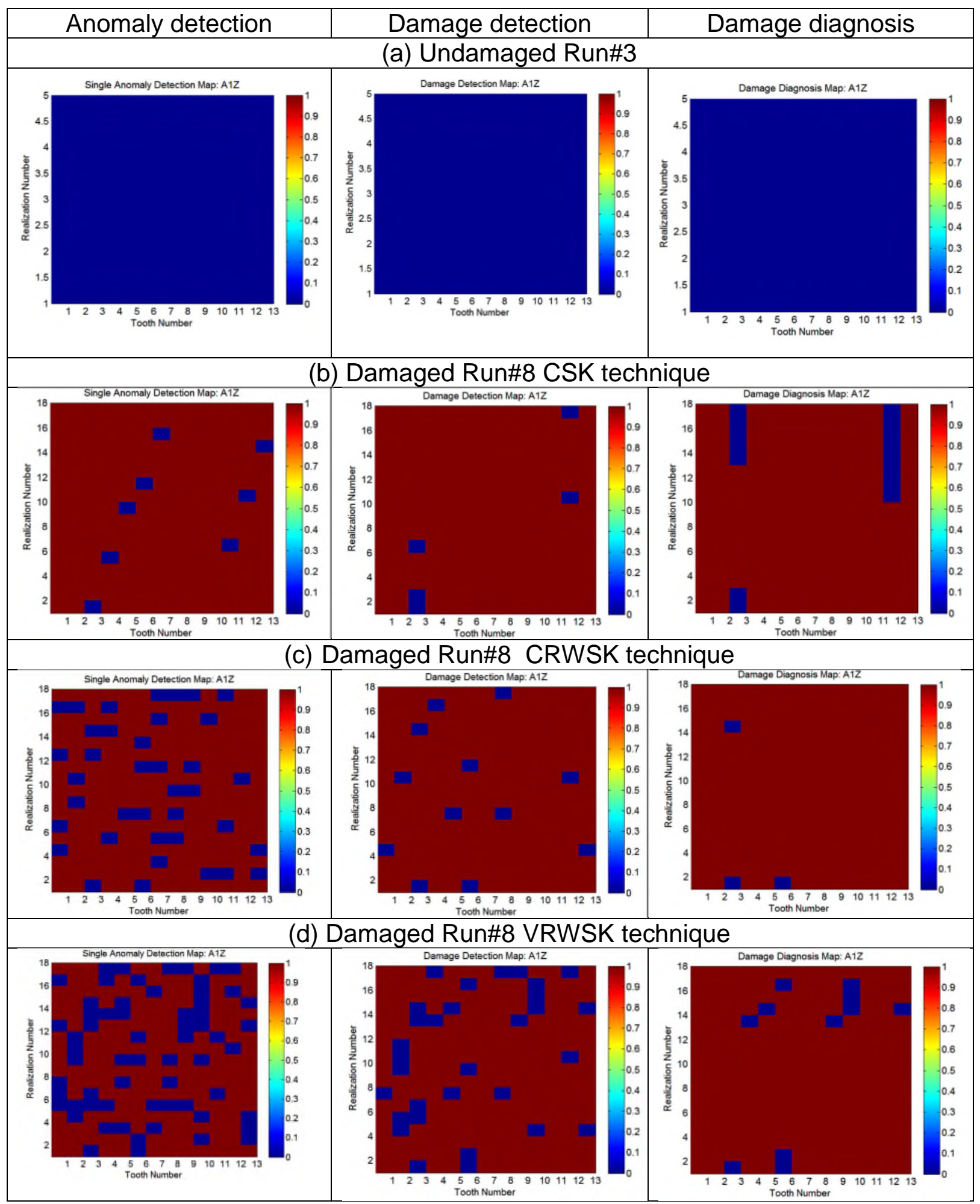

Figure 15 Decision making results with SNR 10dB noise addition for Half mesh SK resolution and

Due to the noise addition the probability of the CSK technique reduced from $100 \%$ to 93.2\%, as shown in Figure 15(b), for the VRWSK technique the probability of correct diagnosis is reduced to $94 \%$ as shown in Figure 15(d). The CRWSK technique has shown $98 \%$ probability of correct diagnosis. Thus it is observed that the error using VRWSK technique is 3 times more than CRWSK technique. The experiments and 
data acquisition tasks are performed at UNIMORE, Italy and the development of the diagnosis technology is done at Cranfield University. UNIMORE did not contribute to the development of the signal processing technology.

\section{Conclusions}

In this paper, two novel industrial studies are presented for developing and testing the wavelet spectral kurtosis technique. A detailed novel comparative study among CSK, VRWSK and CRWSK techniques revealed that, use of CRWSK method shows an essential gain. The Test rig is a two stage industrial gearbox with helical gear pairs. The CRWSK technique could give better performance than the classical SK technique. The toothwise Fisher's Criterion is used to identify the levels of separation between the undamaged and damaged SK features. The gain is estimated as the ratio of Fisher's Criteria obtained using CRWSK technique to that of Fisher's Criterion obtained using CSK technique. The diagnosis decision making technique based on the weighted majority rule is applied to the SK features for final diagnosis decision making. The false alarms that are generated at the anomaly detection stage are eliminated in the damage detection and diagnosis decision making stages.

It is observed that CSK has shown 94\% correct probability of diagnosis for 10dB SNR noise case, while CRWSK technique has shown 98\% probability of diagnosis for same noise level. The error observed in the probability of correct diagnosis is 3 times more for CSK technique than the CRWSK technique. The early diagnosis of the pitting gear faults was successful using the presented SK techniques for the test data sets. Having observed a clear gain for the wavelet spectral kurtosis technique when compared to the classical technique, it is confirmed that the wavelet spectral kurtosis is a reliable tool for diagnosis of gearbox fatigue faults.

\section{Acknowledgments}

The authors acknowledge the financial support of EC IndGear project. 


\section{References}

[1].J. M. Mendel, Tutorial on higher order statistics (spectra) in signal processing and systems theory: theoretical results and some applications, Proceedings of the IEEE, 79(3), pp. 277-305. (1991).

[2]. F. Combet, L. Gelman , G. La Payne, Novel detection of local tooth damage in gears by the wavelet bicoherence, Mechanical Systems and Signal Processing, 26(2012), 218-228.

[3].P. D. McFadden, "Detecting Fatigue Cracks in Gears by Amplitude and Phase Demodulation of the Meshing Vibration," Journal of vibration, acoustics, stress, and reliability in design, vol. 108, no. 2, pp. 165-170, 1986.

[4].S. K. Lee and P. R. White, "The enhancement of impulsive noise and vibration signals for fault detection in rotating and reciprocating machinery," Journal of Sound and Vibration, vol. 217, no. 3, pp. 485-505, Oct. 1998.

[5].Z. Peng and F. Chu, "Application of the wavelet transform in machine condition monitoring and fault diagnostics: a review with bibliography," Mechanical Systems and Signal Processing, vol. 18, no. 2, pp. 199-221, Mar. 2004.

[6].J. Antoni and R.B. Randall. The spectral kurtosis: application to the vibratory surveillance and diagnostics of rotating machines. Mechanical Systems and Signal Processing, 20(2):308 - 331, 2006.

[7]. R. Randall and J. Antoni. Rolling element bearing diagnostics-a tutorial. Mechanical Systems and Signal Processing, 25(2):485-520, 2011.

[8]. Combet F., and Gelman L., An automated methodology for performing time synchronous averaging of a gearbox signal without speed sensor, Mechanical Systems and Signal Processing, Volume 21, Issue 6, August 2007, Pages 2590-2606. 
[9]. F. Combet and L. Gelman, Optimal filtering of gear signals for early damage detection based on spectral kurtosis, Mechanical Systems and Signal Processing, 23(2009) 652-668.

[10]. Yanxue Wang and Ming Liang, An adaptive SK technique and its application for fault detection of rolling element bearings, Mechanical Systems and Signal Processing, 25 (2011), 1750-1764.

[11]. Nader Sawalhi and Robert B. Randall, The application of spectral kurtosis to bearing diagnostics, Proceeding of Acoustics, 393-398, November 2004, Australia.

[12]. Haiyang Liu, Weiguo Huang, Shibin Wang , and Zhongkui Zhu, Adaptive spectral kurtosis filtering based on Morlet wavelet and its application for signal transients detection, Signal Processing , 96 (2014) 118-124.

[13]. L Gelman. B Murray, T H Patel and A Thomson, Novel decision making technique for damage diagnosis, Insight, Vol.55 No 8, 2013.

[14]. J. Antoni. The spectral kurtosis: a useful tool for characterising nonstationary signals. Mechanical Systems and Signal Processing, 20(2):282 307, 2006.

[15]. W. J. Staszewski and G. R. Tomlinson, Application of the wavelet transform to fault detection in a spur gear, Mechanical Systems and Signal Processing (1994) 8 (3), 289-307. 
2016-08-31

Novel spectral kurtosis technology for adaptive vibration condition monitoring of multi-stage gearboxes

\section{Gelman, Leonid}

British Institute of Non-destructive Testing

Gelman, L; Chandra, N Harish et al. Novel spectral kurtosis technology for adaptive vibration condition monitoring of multi-stage gearboxes, Insight - Non-Destructive Testing \& Condition Monitoring, Volume 58, Number 8, August 2016, pp. 409-416

https://doi.org/10.1784/insi.2016.58.8.409

Downloaded from Cranfield Library Services E-Repository 